\title{
Kunden und Dienstleistungsorganisationen - ein Blick in die organisationssoziologische Werkzeugkiste
}

Ursula Holtgrewe

In: Jacobsen, Heike/Voswinkel, Stephan (Hg.) 2005: Der Kunde in der Dienstleistung. Wiesbaden: VS, 37-56

\section{Einführung}

Der vorliegende Beitrag liefert eine Bestandsaufnahme organisationstheoretischer Ansätze und Überlegungen, die sich eignen, den Stellenwert des Kunden und der Kundenbeziehung in Organisationen näher zu beleuchten. Vielfach bleibt in der aktuellen Diskussion über Dienstleistungsarbeit und Dienstleistungsgesellschaften die Ebene der Organisation unterbelichtet. Jedoch sind es Organisationen, die Produkte und Leistungen anbieten, Nachfrage bedienen, Strategien entwickeln, Kunden ansprechen - die also zentral gestalten, wie Dienstleistungsarbeit auszusehen und getan zu werden hat. Die organisationstheoretische Sicht sollte also ein umfassenderes Bild der Dienstleistungsbeziehungen ermöglichen, aber auch umgekehrt wird - so zumindest der Anspruch des Papiers - die Besichtigung geeigneter Organisationstheorien durch die Brille der Dienstleistung die Theorie bereichern.

Strukturell oder auch systemtheoretisch betrachtet, sind Organisationen soziale Gebilde, die Funktionen ausdifferenzieren, sich Zwecke setzen, eigene Regeln und Strukturen aufbauen. Andererseis sind sie offen gegenüber ihren Umwelten, auf Ressourcen und Legitimation aus der Umwelt angewiesen. Handlungstheoretisch betrachtet, sind sie Akteure, die in der Lage sind, diese Umwelten zu ihren Gunsten zu beeinflussen.

Betrachtet man Dienstleistungen, so werden ganz unvermeidlich die 'Schnittstellen' der Organisation als mesosoziologische Analyseebene zwischen der Mikroebene der Interaktion und der Makroebene gesellschaftlicher Ordnungen und Konflikte deutlich, und wir werden uns auf solche Organisationstheorien konzentrieren, die hierzu Aussagen machen. Kap. 2 beginnt mit dem „Klassiker“ der organisationssoziologischen Sicht auf Dienstleistungen: dem „Rationalisierungsdilemma der Angestelltenarbeit“. Auf der Mikro- oder Makroebene sind die Mehrzahl der aktuellen Beiträge zur Dienstleistungsdiskussion zu finden. Insbesondere die Kunden als empirische 
Personen trifft man offensichtlich in der Dienstleistungsinteraktion an (Kap. 3). Wir werden jedoch sehen, dass es sinnvoll ist, sie auch und zentral als Konstrukte von Organisationen zu beleuchten. Das ist in einem kognitiven Sinn fast schon trivial - aber Organisationen entwickeln auch Strategien (Kap. 4), um diskursiv und praktisch 'den' Kunden zu beeinflussen, zu positionieren, oder je nach theoretischem Standort gar als Subjekt zu konstituieren. Auf der Makroebene wird über Dienstleistungsgesellschaften diskutiert: Über die Reichweite und Folgen der Tertiarisierung (Baethge/Wilkens 2001), die Konvergenz oder Pfadabhängigkeit von Dienstleistungsgesellschaften (Häußermann/Siebel 1995), oder die mcdonaldisierte Gesellschaft (Ritzer 1996). Hier können Organisationstheorien anschließen, die Organisationen als sozial eingebettet verstehen: in soziale Felder, institutionelle Ensembles oder Regimes (Kap. 5) und in Kulturen und Gesellschaften, die diese wiederum prägen. Zwischen strategischem Handeln und sozialer Einbettung entstehen nicht intendierte Folgen und Wechselwirkungen (Kap. 6) - und hier sind nicht nur Dienstleistungsorganisationen, sondern auch ihre Kunden positioniert und positionieren sie sich selbst (Kap. 7).

Ein geschlossenes organisationstheoretisches Modell wird daraus nicht entstehen. Der theoretische Standort der Autorin ist eklektizistisch aus Überzeugung: Ich folge Ortmann, Sydow und Windeler (1997), die die Giddenssche Strukturationstheorie als eine Art Gerüst vorschlagen, in das man andere Organisationstheorien und Perspektiven einhängen kann. Dazu ist es notwendig, diese unter der Perspektive des Handelns und Konstruierens zu lesen, das Strukturen bildet, die wiederum Handeln ermöglichen und restringieren usw. Jedoch wird dem Gegenstand Dienstleistung entsprechend der Akzent stärker auf das sozial Konstruktive dieser Prozesse gesetzt (Kap. 8): Auf das aktive Herstellen, Verändern und Zusammenfügen - eine symbolischinteraktionistische Giddens-Lesart bildet den Hintergrund für die vorliegenden Ausführungen.

\section{Das Rationalisierungsdilemma der Angestelltenarbeit revisited: Dienstleistung und Ungewissheit}

Als Ausgangspunkt einer Besichtigung von Organisationstheorien bieten sich die geradezu klassischen Arbeiten an, die Ulrike und Johannes Berger und Claus Offe in den frühen 80er Jahren zum Rationalisierungsdilemma der 
Angestelltenarbeit vorgelegt haben (Berger/Offe 1981; Offe 1984). Diese AutorInnen haben bekanntlich das organisationstheoretische Konzept des technologischen Kerns und der Gewährleistungsfunktionen einer Organisation (Thompson 1967) aufgegriffen und auf Arbeit einerseits, Gesellschaft andererseits ausgedehnt. Die Besonderheiten der Dienstleistungsarbeit und des Dienstleistungssektors machten sie in deren Gewährleistungsfunktion gegenüber der eigentlichen, produktiven Arbeit aus. Der Dienstleistung obliege die „Gewährleistung [der] institutionellen Ordnung und der übrigen funktionalen Voraussetzungen dafür, dass Produktionsarbeit vonstatten gehen kann“ (Berger/Offe 1981: 41) in Organisationen und Gesellschaften.

Dazu muss man sich das klassische Modell einer fordistischen Produktionsorganisation vor Augen führen: Hier wird der produktive Kern zur Steigerung seiner Effizienz von der turbulenten Umwelt abgepuffert, indem Organisationen ihn mit spezialisierten Einheiten umgeben, die die entsprechenden Umweltimpulse bearbeiten, filtern und übersetzen. Mit der Umwelt der Organisation befassen sich dann verschiedene Arten von Grenzstellen (wie Ein- und Verkaufsabteilungen, Personalabteilungen, Pressestellen usw.), auf dass der Kern in Ruhe arbeiten kann. Für die Gewährleistungsarbeit müssen demzufolge, weil sie es bereits mit turbulenten Umwelten und Unvorhersehbarkeiten zu tun hat, andere Kennzeichen gelten als die der ökonomischen Effizienz: Kriterien der Effektivität, der Sicherung langfristiger Bestandserhaltung und der Bewirtschaftung von organisationellen Reservekapazitäten (= slack) angesichts einer strukturellen Unsicherheit über Inputs und Outputs. Dies sind klassische Konzepte der Organisationssoziologie: Begrenzte Rationalität (Simon 1957), Logiken der Angemessenheit und der Legitimation einerseits (March/Olsen 1976; 1989), der strategischen Einflussnahme auf die Umwelt und der ,politischen“ Aushandlung andererseits. Sie eignen sich $\mathrm{m}$. E. in der Tat, die Lücken zu füllen, die eine industriesoziologische Betrachtung der Dienstleistung lässt - und nicht umsonst sind ja vielfach Dienstleistungs-Organisationen wie Verwaltungen, Krankenhäuser, Hochschulen usw. Gegenstand der Organisationssoziologie.

Das Modell einer Trennung von Produktions- und Marktökonomie bei Berger, Berger und Offe ist nun vielfach dafür kritisiert worden, dass es an einer industrialistischen Vorstellung von 'richtiger' Arbeit und einer Unterscheidung von eigentlich produktiver und unproduktiver Arbeit hängt (Gottschall 2001) und es ist zum Zeitpunkt der Überlegungen dieser AutorInnen auch schon fast obsolet gewesen: Anfang der 80er Jahre zeichnete sich ja bereits ab, dass 
Unternehmen begannen, sich zum Markt zu öffnen, Umweltturbulenzen „hereinzulassen“ statt sie abzupuffern ( z. B. Brose 1982). Mir scheint aber, dass das „Rationalisierungsdilemma der Angestelltenarbeit“ zwischen Effizienz und Flexibilität, Grenzöffnung und -schließung für Organisationen (also dort, wo es zu Hause ist) durchaus gut erfasst ist. Nur: Es ist dann nicht mehr in der schlichten Ausdifferenzierung von Kern- und Gewährleistungsfunktionen zu bearbeiten, wenn Organisationen in turbulentere Umwelten geraten, und wenn sie ihrerseits gerade aus dem Durchschlagen der Umweltturbulenzen auf den 'Kern' Effizienzgewinne zu ziehen suchen.

Konstitutiv für die Dienstleistung bleibt jedoch die strukturelle Input- und Output-Ungewissheit: Wie man so sagt, ist die Hälfte allen Geldes, das Unternehmen für Werbung ausgeben, rausgeschmissen - wenn sie nur wüssten, welche Hälfte. Betriebswirtschaftlich betrachtet, liegt das daran, dass Dienstleistungen „unter Einsatz externer Produktionsfaktoren für den fremden Bedarf produzierte immaterielle Wirtschaftsgüter" sind (Maleri 1998: 123, zit. nach Weihrich/Dunkel 2003: 761): Das heißt, die Kundin muss, um eine Dienstleistung in Anspruch zu nehmen, eigene Ressourcen und/oder eigene Kooperationsbereitschaft einsetzen. Daraus ergibt sich, dass Dienstleistungen erst im Verlauf der Dienstleistung (interaktiv) spezifiziert werden - und diese Spezifizierung selbst kann (als Beratung) ein wichtiger Teil der Dienstleistung sein.

Halten wir also die Unterdefiniertheit und Offenheit von Dienstleistungen und deren potenziell selbstbezüglich ausdehnbaren Charakter (Beratung über Beratung ...) fest. Dienstleistungen werden erst im Verlauf spezifiziert. Für Organisationen bedeutet das: Sie haben es ganz offensichtlich im Produktionsprozess selbst mit der Definition ihrer Leistungen und Produkte und damit mit dem Ziehen von Grenzen zu tun. Diese vorläufige Definition von Dienstleistung und ihre Abgrenzung zur Produktion ist auf jeden Fall durchlässig, und das wird sie umso mehr, wenn sich auch die Produktion materieller Güter über deren Wissensintensität, Innovativität, Kundenbezogenheit usw. quasi dienstleistifiziert.

Die Unterdefiniertheit und strukturelle Input/Output-Ungewissheit von Dienstleistungen setzt der Logik der Effizienz immer wieder neue Grenzen und bildet auf der Organisationsebene das Einfallstor für Spielräume und Kontingenzen, für empirisch vielfältige und heterogene Ausfüllungen von oben und von unten (Holtgrewe/Kerst 2002a; b). „Oben“ meint dabei die Ebene der Institutionen und der Gesellschaft, der verfügbaren Ressourcen, Regeln, 
Regulierungen, Normen und kulturellen Interpretationsrahmen, die das Agieren von Organisationen einbetten. „Unten“ meint die Ebene der Interaktionen, in denen zwischen Arbeitenden in der Organisation und KundInnen Dienste und Leistungen ausgehandelt, spezifiziert und bewertet werden. In den folgenden Abschnitten gehen wir der Frage nach, wie diese Ebenen wiederum durch Organisationen enacted und gestaltet werden (Jepperson 1991) - im doppelten Sinne von Aktualisierung und Strategie - und mit welchen theoretischen Instrumenten man diese Gestaltungsprozesse untersuchen kann.

\section{Organisation und Interaktion}

\section{$3.1 \quad$ Grenzstellen}

Betrachtet man die Ebene der Interaktion aus organisationstheoretischer Sicht, so finden Interaktionen zwischen Dienstleisterinnen und Kunden an den Grenzstellen von Organisationen, „on the frontline“ (Frenkel et al. 1999) statt. Grenzstellen sind die spezialisierten Einheiten einer Organisation, die diese mit ihren Umwelten verbinden: Callcenter, Verkaufstheken, Bankschalter, Bürgerbüros usw. An der Grenzstelle wird das strukturelle Problem von Organisationen prozessiert, ihr Flexibilitätsdilemma (Kühl 1995): Öffnung und Schließung, Reaktionsfähigkeit und Stabilisierung ins Werk zu setzen (Tacke 1997).

Die Rationalisierung von Grenzstellenarbeit im Sinne der Effizienzsteigerung ist daher problematisch (Holtgrewe/Kerst 2002b). Sie richtet sich auf nur eine Seite des Dilemmas, die der Schließung. Im mcdonaldistischen Produktionsmodell bekommt der Versuch der Rationalisierung systemischen Charakter (Voswinkel 2000): Dann werden nicht nur Prozesse, sondern auch Produkte, Arbeits- und Kundenbeziehungen - unter spezifischen kulturellen und institutionellen Voraussetzungen - neu gestaltet und standardisiert. Aber selbst McDonald's bastelt ja immer wieder am reengineering seiner Angebote, stellt Menüs zusammen und veranlasst seine Beschäftigten zum cross selling von Apfeltaschen zum Hamburger usw. - auch dezidierte Standard-Dienstleister suchen also die Möglichkeiten zu nutzen, die die Interaktion mit den Kunden zur Spezifizierung und Expansion der Dienstleistung bietet. 


\subsection{Macht und Kontrolle an der Grenzstelle}

Mit der Sicht auf die Grenzstelle - ein zunächst systemtheoretisches Konzept ist es auch möglich, einen Blick auf Macht und Herrschaft in Organisationen zu werfen. Für die Organisation ist der Dienstleister ihr 'Agent', der, mit notwendigen Handlungsspielräumen versehen, ihre Vorgaben in Auseinandersetzung mit der Umwelt um- und durchzusetzen sucht. Eben darin, im Handeln und Interagieren auf vorgeschobenem Posten im Namen der Organisation, besteht interaktive Dienstleistungsarbeit - mitsamt den bekannten principal-agent-Problemen, dass diese Agentin auf eigene Rechnung arbeitet, eigene Interessen über die der Organisation stellt. All dies bewegt sich jedoch im Rahmen von Arbeitsverträgen und betrieblicher Herrschaft.

Die Kundin gehört jedoch zur Umwelt oder zum Publikum (Stichweh 1988), sie ist nicht von der Organisation angestellt, das heißt, die Organisation kann ihr erst einmal keine Weisungen erteilen. Die Kundin zur Kooperation und zur Abstimmung ihrer Wünsche auf das Angebot der Organisation zu bewegen, muss auf Märkten mittelbarer funktionieren. Vieles davon geschieht eben in der Interaktion. Diese findet in dem berühmten Kontrolldreieck statt (Leidner 1996: 38ff.), in dem sich alle Arten von Allianzen finden lassen: Die Organisation sucht mittels der Kundin den Grenzstellen-Dienstleister zu kontrollieren, z. B. über Kundenzufriedenheitsbefragungen (s. Fuller/Smith 1996). Organisation und Dienstleister kontrollieren einträchtig die Kundin. Oder Kundin und Dienstleister verbünden sich „gegen“ die Organisation: Der Dienstleister lotet etwa für die Kundin Kulanzspielräume aus.

Aber Organisationen wären keine Organisationen, wenn sie nicht vor und nach der Interaktion noch genuin organisationelle Mittel zum Einsatz brächten: Die Gestaltung eines attraktiven Angebots, den Einfluss auf die Präferenzen der Kundin (Werbung, symbolische Kommunikation), und die Gestaltung der Organisation selbst, ihrer Zugangswege, Kontaktmöglichkeiten und Grenzstellen. Ganz technisch-sachlich-materiell kann ja die Kundin genötigt werden, Einkaufswagen nicht nur zu benutzen, sondern auch zurückzubringen, sich der Telefonnummer des Callcenters zu bedienen, sich an Öffnungszeiten zu halten usw. Hier bietet gerade die Information- und Kommunikationstechnik (aber auch die Architektur) vielfältige Möglichkeiten, materielle und symbolische Gestaltung in einem zu betreiben und Alternativen für die Kundin auszuschließen bis hin zur Selbstbedienung. 
So betrachtet, sind wir beim alten Thema von Colemans „asymmetrischer Gesellschaft" (Coleman 1986): Organisationen auf unvollkommenen Märkten (der Normalfall) haben per Definition mehr Macht, mehr Information und mehr Dispositionsspielräume als der gemeine Individualkunde, und Gesellschaften müssen die Begegnungen solch unterschiedlicher, individueller und kollektiver Akteure regeln. Andererseits aber stoßen gerade die räumlich-technischen Arrangements der Selbstbedienung und standardisierten Dienstleistung durchaus auf ein eigenes Interesse der KundInnen an Bewegungsfreiheit, Selbstbestimmung und Nicht-Interaktion (Voswinkel 2000). In solchen Fällen wird ganz kollusiv Kunden-Autonomie gespielt - wobei in die Genese solcher Autonomie-Interessen der KundInnen selbstverständlich vorgängige Organisations-Interaktions-Erfahrungen und entsprechende Erwartungen eingehen.

\subsection{Macht und Diskurs}

Am Punkt der Konstitution von Beherrschungs- oder Autonomie-Interessen wird das Bild des Kontrolldreiecks ein Stück unterkomplex. Es impliziert eine Sicht auf Macht als Nullsummenspiel (wer kontrolliert wen?). Es lohnt sich, hier andere Machtbegriffe in Anschlag zu bringen, die einbeziehen, dass Macht auf mehreren Ebenen operiert: Von der Verfügung über Ressourcen und Regeln (Giddens 1988) oder über Handlungsrechte, die konflikthaft oder konsensuell übertragen werden können (Coleman 1991) bis zur Verfügung über die Beteiligung an Entscheidungen und die Verteilung von Zurechnungen, über Problemdefinitionen und darüber, zu definieren, was als Ressource gilt und wie weit eine Regel reicht (Friedberg 1995; Hardy/Leiba-O‘Sullivan 1998). Und diese Ebenen der Macht und ihrer Ausübung verteilen sich wiederum in je spezifischer Weise auf die Interaktion, die Gestaltung der technisch-materiellen Ausstattung und der symbolischen Selbstdarstellung durch die Organisationen, und auf die institutionalisierten und kulturell abgebildeten Erwartungen der Akteure.

Noch weiter gehen die von Foucault inspirierten Überlegungen aus den „Critical Management Studies“ und der postmodernen Organisationstheorie. Sie nehmen an dass Organisationen selbst Beschäftigte wie auch KundInnen als Subjekte konstituieren. Für Dienstleistungen ist diese Sichtweise besonders plausibel, weil hier ja in der Tat die Kunden über diskursive und symbolische 
Mechanismen zum Kooperieren gebracht werden (Rieder 2001). Institutionen und Organisationen statten so betrachtet die Individuen mit materiellen und symbolischen Ressourcen aus, die ihnen erst einen Sinn von Autonomie verleihen. Der Verkauf von Finanzdienstleistungen etwa knüpft an Lebenspläne, Phantasien vom Reichtum und Wünsche nach Autonomie an - sowie an die entsprechenden Ängste vor Armut und Abhängigkeit (Knights/Morgan 1994). Dass Verkauf und Beratung bei Finanzdienstleistungen funktionieren, fordert dann auch den Kunden entsprechende Biographisierungsleistungen ab. Das nimmt zu, wenn soziale Absicherung stärker über Finanzmärkte als über Sozialversicherungen gewährleistet wird. Organisationen produzieren in der Interaktion mit dem Kunden diesen selbst als selbstverantwortliches Subjekt, das sich seine Entscheidungen zurechnen muss.

Auch auf der Ebene der Diskurse aber greifen Herrschafts- und Kontrolldialektiken. Korczynski (2002) argumentiert, dass Unternehmen gegenüber ihren Kunden die Konsumwelt nicht allein zu rationalisieren, sondern wieder zu „verzaubern“ suchen, und dass zu dieser Verführung und Verzauberung gerade die Fiktion des selbstbestimmten freien, königlichen Konsumenten gehört. Diese aber kann sich in konfliktträchtiger Weise verselbstständigen: Wer als mutmaßlicher Souverän verführt wird, die oder der unterwirft sich vielleicht willig und regressiv der Organisation und generalisiert sein Vertrauen, dass diese ihm alle Bedürfnisse erfüllt - aber gleichzeitig mögen seine Ansprüche auf umfassende Versorgung, Verfügbarkeit und umfassendes Funktionieren auf ein hoch enttäuschungsanfälliges Maß steigen (vgl. Holtgrewe/Voswinkel 2002). Die Ausdehnung des Reise- und Verbraucherrechts und die vielfältigen und seltsamen Rechtsstreitigkeiten um Werbeaussagen von Reiseprospekten und Vertragsinhalte machen dies deutlich.

Vielleicht aber mutiert der Souverän angesichts der Vielzahl der Verzauberungen auch zum smart shopper, der die Verzauberungsversuche geschmäcklerisch zur Kenntnis nimmt und ganz strategisch sucht, wer ihm denn was zu bieten hat. Das freilich spricht noch nicht gegen die Wirksamkeit diskursiver Subjektkonstitution, wie spätestens die Media-Markt-Werbung „Ich bin ja nicht blöd“ deutlich macht. Der „Durchblick“, der dem smart shopper gegenüber Werbung, Warenästhetik und Organisationsstrategie unterstellt wird, mag ihn umso tiefer in die Diskurse des Marktes und die Fiktionen der Konsumentensouveränität verstricken (vgl. Fleming/Spicer 2003 für Subjektivität in Arbeitsorganisationen). 
Ob die diskursive Konstitution des smart shopper aber einen Sozialcharakter hervorbringt, scheint mir fraglich. Er ist zunächst ein Artefakt organisationeller Umweltmodellierung sozusagen zweiter Ordnung. Marktforschungsunternehmen, Trendbüros und Werbeagenturen, also Spezialdienstleister für organisationelle Umweltbeobachtung reflektieren hier auf die dialectic of control und die nichtintendierten und aggregierten Folgen organisationaler Strategien.

\section{Organisationen und Umwelten: Strukturen und Strategien}

Organisationen entwickeln in der Gestaltung der Grenzstelle auch genuin organisationelle Methoden der Rationalisierung - die den AgentInnen dann als restringierende Vorgaben oder auch als ermöglichende und entlastende Werkzeuge und Ressourcen gegenübertreten. Wenn also Organisationen ihre Kunden beobachten und modellieren, so hat dies nicht nur kognitiven Charakter. Sie tun es auch ganz strategisch. Kundensegmentierung etwa ist ein gewichtiges Werkzeug der Dienstleistungsrationalisierung. Organisationen konzentrieren ihren schlecht kalkulierbaren, beratungsintensiven Aufwand an den Grenzstellen auf die Kunden, bei denen es sich lohnt, von denen sie die höchsten Deckungsbeiträge zu erwarten haben. Die Kunden mit den kleineren Umsätzen werden auf Standardprodukte und technisierte Zugangswege verwiesen. Wir haben das bei der Telekom beobachten können (Blutner/Brose/Holtgrewe 2000), Rosemary Batt in den USA desgleichen bei Telekommunikations- und Finanzdienstleistungen (2000), und Gerd Möll (2003) im Handel. Ebenso wie bei der mcdonaldistischen Standardisierung wird hier die umfassend konstruktive Seite organisationellen Agierens deutlich: Das Ineinandergreifen von Grenzstellen-, Produkt-/Leistungsgestaltung und Kunden-Modellierung, die alle immer wieder flexibel auf einander abgestimmt werden. Nebenbei vervielfältigen sich die Grenzstellen und Dienstleistungen: Spezialdienstleister für Beratung, Marktforschung oder Zusatz-Services kommen ins Spiel, und die Verteilung und Kombination des strategischen Wissens über die Kunden wird zum Gegenstand organisationeller Vernetzung weit über die Interaktion hinaus (Möll 2003).

Auch die Kundensegmentierung aber erweist sich nicht als one best way der Dienstleistungsrationalisierung - von ihren sozial problematischen Folgen abgesehen, Beratung bei den KundInnen zu konzentrieren, die sie nicht am 
nötigsten haben (D'Alessio/Oberbeck 1998). Hier sind die Dienstleister bei einem keineswegs allein effizienzgetriebenen Hin-und-Her zu beobachten: Vom Sortieren nach Umsatzgröße zum Sortieren nach technischer Ausstattung oder Branche im Geschäftskundenmanagement der Telekom, vom ausgelagerten Private Banking zurück in die Filialen bei der Deutschen Bank, vom ausgesourcten Direktbanking (das sich allerdings durchaus nicht an die ärmsten Kunden richtete) zum Multikanalvertrieb (Regini u. a. 1999) usw. - weil Kostensenkung wieder Dienstleistungsbedarf generiert, weil die Kundin „qualifiziert" werden muss, und weil Angebotsstandardisierung auch die Möglichkeiten der Interaktion verschenkt, neuen Umsatz zu generieren.

Ein weiterer aussichtsreicher organisationeller Weg der Bewegung im Flexibilitätsdilemma besteht in der Heuchelei. Unter OrganisationsforscherInnen meint das nichts moralisch Verwerfliches, sondern die organisationsspezifische Möglichkeit, „Reden“ und „Handeln“ auszudifferenzieren, die symbolische Kommunikation vom realen Entscheiden und der Leistungserstellung zu entkoppeln (Brunsson 1989). Unter uno-actuBedingungen ist das in der Interaktion jedoch nur begrenzt möglich: Wenn nicht die Organisation insgesamt und arbeitsteilig, sondern wenn an Grenzstellen Individuen lügen und heucheln müssen (z. B. über Lieferfristen etc.), ist das riskant, weil für diese ja erst einmal Normen der Wahrhaftigkeit gelten. Der personelle Vertrauensbonus, den Interaktionen der Organisation verleihen (sollen), kann dann sehr schnell implodieren. Unter rationalisierten Bedingungen und bei der Vervielfältigung von Grenzstellen und Vertriebswegen aber eröffnen sich Möglichkeiten: In der Vermittlung anderer Dienstleistungen etwa, oder in der symbolischen Darstellung von Kundenorientierung (weiß man etwa, was mit den Ergebnissen all der Zufriedenheitsbefragungen geschieht?) Auch hier spielen sich wohlgemerkt die Standards von wahrheitsgetreuer Darstellung sowohl interaktiv als auch institutionell ein: Kompetente Kunden und Dienstleister können zwischen Wahrhaftigkeit und beidseitig strategischer Interaktion manövrieren und mitunter spielen. Den Rahmen dafür bilden Institutionen, die auch mächtigen Organisationen Erwartungen, Regeln und Normen auf die Tagesordnung setzen.

\section{Organisationen, Institutionen und Legitimation}

\subsection{Der Neoinstitutionalismus}

10 
Die Einbettung von Organisationen in ihre sozialen Umwelten wirkt als Gegengewicht zum Einfluss, den sie strategisch auf ihre Umwelten ausüben. Für diese Zusammenhänge und für die Aktualisierung von anderen Logiken als solchen der Effizienz ist der Neoinstitutionalismus die erste theoretische Adresse. Seine VertreterInnen ${ }^{1}$ betonen, wie sehr organisationale Strukturen und Praxen sich an der Legitimation ausrichten, die Organisationen in ihren jeweiligen Feldern und Handlungskontexten brauchen - und die auch die Kriterien und Ausrichtungen wirtschaftlicher Rationalität selbst prägen. Die NeoinstitutionalistInnen verweisen gegenüber der wirtschaftlichen Effizienz auf die Bedeutung von Umwelterwartungen, situierten Angemessenheitsorientierungen und Routinen und neuerdings auf die soziale Konstruiertheit auch mutmaßlich „harter“ ökonomischer Leistungs- und Erfolgskriterien (Quack/Morgan/Whitley 2000). Auch Rationalität selbst ist eine solche institutionalisierte Umwelterwartung (Meyer/Rowan 1977), ein Mythos, der aber sehr reale Wirkungen hat.

Umso mehr dann, wenn die „harten“ Kriterien für Effizienz fehlen, wird also die strukturelle Input- und Output-Ungewissheit zum Einfallstor für Angemessenheits- und Legitimationslogiken (so auch Berger/Offe 1981). Und diese Angemessenheitslogiken orientieren sich entweder an den Erwartungen der Umwelt oder an dem, was die anderen tun. Seit den Arbeiten von DiMaggio und Powell (1991) ist hier von der Isomorphie die Rede: Über staatlichen Zwang, normativen Druck etwa von Professionen oder über das Nachahmen von Wettbewerbern, die man als erfolgreich betrachtet, gleichen Organisationen in einem institutionellen Feld ihre Strukturen und Praxen aneinander an. Das erklärt etwa das Diffundieren von Reorganisationskonzepten oder Managementmoden, und es lässt auch die Akteure solcher Diffusionsprozesse in den Blick treten. Unternehmensberatungen, Zertifizierungsagenturen und andere Unternehmensdienstleister oder Technologiehersteller können als Promotoren der Isomorphie ins Auge gefasst werden, wiewohl die tatsächliche Angleichung oder die Persistenz historischer, kultureller, regionaler oder anderer Unterschiede eine empirische Frage ist.

Empirisch konzentrierten sich die AutorInnen des Neoinstitutionalismus zunächst auf Organisationen aus dem Bildungs- und Kultursektor. Hier spielt gegenüber Wettbewerb und Effizienz die Legitimität die größere Rolle. Ergänzen und festhalten kann man dazu, dass solche Organisationen Legitimität und sozialen Sinn ja nicht nur als Bestandsvoraussetzung brauchen, sondern 
ihrerseits ganz offensichtlich produzieren. In späteren Arbeiten wurde jedoch deutlich, dass auch das Wirtschaftssystem mit seinen spezifischen Effizienzund Wettbewerbsmustern gesellschaftlich institutionalisiert ist: „Die Stabilisierung und Nicht-Hinterfragung derartiger Bereiche gilt vielmehr selbst als Resultat institutioneller Orientierungen; auch sie ist legitimatorisch, symbolisch und sinnhaft begründet" (Hasse/Krücken 1999: 40), und: Auch die Wirtschaftsorganisationen produzieren Sinn, Symbolik und Legitimation strategisch oder versehentlich mit. Das wird dann überdeutlich, wenn die Semantik des Kunden in öffentliche Verwaltungen, Hochschulen oder Krankenhäuser diffundiert und zur Legitimationsressource für alle möglichen strukturellen und symbolischen Reorganisationsschritte oder auch für entsprechende Forderungen der „KundInnen“ oder des politischen Systems wird.

Wichtig ist, dass auch bei marktförmig erbrachten Dienstleistungen, deren Ungewissheiten und Risiken von ,unten“ durch Interaktionen aufgefüllt werden, dies „oben“ durch Institutionen geschieht - nicht nur durch rechtliche Regulierungen und staatlichen Zwang, sondern auch durch institutionalisierte Erwartungen und Legitimationserfordernisse. Hier werden formelle oder informelle Standards für Leistungsqualität und -umfang, für symbolische Repräsentation und Transparenz gesetzt. Was also an Service "zum Service“ gehört und pauschal eingerechnet wird und auf welche Teile der Dienstleistung ein Preisschild geklebt wird, wird weder ausschließlich in der Interaktion ausgehandelt, noch allein von der Organisation strategisch gestaltet. Aushandlung und Gestaltung werden gerahmt von institutionalisierten Erwartungen der Umwelt - die jedoch Organisationen und Interaktionsteilnehmer immer wieder einerseits reproduzieren, andererseits transformieren.

Der Punkt der Regulierung ist in den letzten Jahren besonders in internationalen Vergleichen bestimmter Dienstleistungs- und Infrastrukturbranchen (wie Finanzdienstleistungen, Telekommunikation, Verkehr oder Einzelhandel) deutlich geworden (z. B. die Beiträge in Quack/Morgan/Whitley 2000; Rudolph 2001). Insbesondere Quack und Morgan haben herausgearbeitet, wie heterogen und in institutionelle Kontexte eingebettet auch die mutmaßlich harten ökonomischen Leistungs- und Erfolgskriterien sind. Weniger erforscht sind die weicheren, normativen und kulturellen Rahmungen und Erwartungen, die Gesellschaften, Öffentlichkeiten und KundInnen an die Qualität, den Umfang und die Organisation von 
Dienstleistungen stellen. Hier sind noch vielfältige Verbindungen von Konsumund Organisationssoziologie denkbar und aussichtsreich.

\subsection{Neoinstitutionalismus und Strategie: wechselseitige Spezifikationen}

Es sollte klar geworden sein, dass sich die Sicht auf die Strategien und Konstruktionen von Organisationen und die Betonung der institutionalisierten Erwartungen und Legitimationserfordernisse ergänzen - im Sinne einer strukturationstheoretischen Wechselwirkung. Was wir sozial unter Dienst, Leistung oder Arbeit verstehen, was wir erwarten und womit wir als KundInnen „Zufrieden“ sind, das ist einerseits ein Aggregat gesellschaftlich institutionalisierter Erwartungen, Normen und Verkehrsformen, andererseits aber auch Produkt der Dienste, Leistungen usw. und der uno actu mitgelieferten Symbolik und Inszenierung von Organisationen. Auf diese rekursive Spezifikation dessen, was ökonomische Rationalität und Rationalisierung - in unserem Fall: Dienst und Leistung - eben keineswegs von vornherein „sind“, sondern wie sie immer erst im Vollzug hergestellt werden, macht immer wieder Günther Ortmann aufmerksam (2003).

Dieser Punkt ist insbesondere hervorzuheben, wenn man vermeiden will, sich mit den Konzepten des Neoinstitutionalismus ein „oversocialized“ Verständnis von Organisationen als Konformisten gegenüber sozial institutionalisierten Erwartungen einzuhandeln - und damit kommt man ja schon bei Individuen nicht weit. Auch das Wechselspiel zwischen Organisationen und Institutionen, die sie formell oder informell regulieren, verläuft rekursiv (Ortmann/Zimmer 2001): Organisationen suchen die Erwartungen und Regulierungen ihres institutionellen Feldes selbst zu beeinflussen, und dafür gibt es wiederum Spezialdienstleister und entsprechende Dreiecks- und Netzwerkbeziehungen: Werbung, Lobbying, die aus der Politikwissenschaft vielfach bekannten Verflechtungen zwischen Regulierern und Regulierten sind Beispiele.

Hier nähern wir also die Sicht des Neoinstitutionalismus der akteurorientierten, politischen und konstruktiven Sicht etwa von Friedberg an, in der institutionalisierte, formelle wie informelle Regeln in den respektiven Handlungsarenen immer gleichzeitig eingehalten und übertreten werden (Friedberg 1995: 213). Die Akteure versuchen immer wieder, Ungewissheiten zu kontrollieren und Unausgewogenheiten in ihren Beziehungen zu ändern, sie 
handeln situiert, manövrieren und folgen eigenen Impulsen. Sie versuchen, die „Prägnanz des formalen Rahmens nach und nach anzuknabbern“ (145) und dies auch mit emergierenden Regeln und Strukturen zu tun.

\section{Organisationen und Kunden in Gesellschaft: Risiken und Nebenwirkungen}

Aber all die Strategie hat natürlich ihre Grenzen. Es gibt Emergenzeffekte und nicht intendierte Folgen, und diese werden auf der gesellschaftlichen Ebene artikuliert. Hier stehen den mehr oder weniger marktrationalen oder gebundenverzauberten Kunden verschiedene Advokaten und Öffentlichkeiten zur Seite und die demonstrative Wahrnehmung von Kunden- oder Konsumenteninteressen wird ja von Medien wiederum als probates Mittel der Kunden- bzw. „Leser-Blatt-Bindung“, wie es bei Zeitungen heißt, eingesetzt. Die skizzierte Kundensegmentierung etwa stößt - jedenfalls in Deutschland vielfach auf öffentliche Skepsis. Dass hier Unternehmen ungleiche Ressourcenausstattungen ihrer Kunden strategisch zu nutzen suchen, gilt nicht ohne weiteres als legitim. Als die Deutsche Bank ihre kleineren und ärmeren Kunden zur Bank24 zu schieben suchte, die zuerst als Direktbank gegründet worden war, erhob sich der Vorwurf der „Zweiklassengesellschaft“ (Die Zeit 30. März 2000). Das jedoch gilt nicht überall. Außerhalb von Kreuzberger Autonomenzirkeln finden sich wenig öffentlich wahrnehmbare Einwände gegen Luxusrestaurants, und bei Transportdienstleistungen hat die erste, zweite oder Business Class Tradition. ${ }^{2}$ Ich vermute, dass Kritik dort laut wird, wo solche Strategien als Kreation statt als Ausdruck sozialer Ungleichheit wahrgenommen werden.

Nicht nur auf der Seite der Arbeit - auf die ich hier nicht weiter eingehe ${ }^{3}$ sondern auch auf der des Konsums also schließen Dienstleistungen an die Sozialstruktur an; sie nutzen sie, beuten sie aus und gestalten sie. Die Herstellung sozialer Distinktion ist Gegenstand von Luxus-Dienstleistungen, und Dienstleistungen eignen sich besonders gut für den demonstrativen Konsum (Veblen 1986). Aber auch ein großer Teil der gesellschaftlich für wichtig gehaltenen „Daseinsvorsorge“ für alle wird in Gestalt von Dienstleistungen erbracht. Je nach dem wohlfahrtsstaatlichen und dem Geschlechterregime (Häußermann/Siebel 1995, Pfau-Effinger 2001) einer Gesellschaft ist dafür der öffentliche oder mischwirtschaftliche Sektor zuständig, der Markt mit mehr oder 
weniger staatlicher Umverteilung, oder die Frauen in den Privathaushalten - mit entsprechenden Rückwirkungen auf ihre Arbeitsmarktchancen und wiederum auf die Nutzung von Dienstleistungen (Geissler 2002; Meyer 2002). Öffentliche, marktförmige und private Dienstleistungen greifen somit subsidiär, kompensatorisch oder komplementär ineinander. Bekanntlich wandern aktuell Marktelemente in die ehedem öffentlichen Sektoren wohlfahrtsstaatlicher Dienstleistung ein. Der öffentliche Sektor reicht die Zuständigkeit für die Gewährleistung sozialer Mindestversorgung an den Markt weiter, positioniert BürgerInnen (mehr oder minder heuchlerisch) als Kunden und nimmt Ungleichheit in Kauf.

Die Frage ist, wie sich angesichts der Verschiebung und Vermischung von „Kunden“-, Klienten- und Bürgerrollen Kundenbeziehungen und -erwartungen verändern, und um dieser Frage zwischen Markt und Gesellschaft nachzugehen, muss man die nicht intendierten Folgen organisationeller Strategien und der Kundenerwartungen, die sie wecken, enbeziehen. Sie reichen vom Gefangenendilemma für Organisationen bis zur gesellschaftlichen Verarbeitung und zum Wandel sozialer Normen und kultureller Bilder - und diese sind ja, wie gesehen, nicht nur „Input" von Umweltanforderungen an die Organisation, sondern auch strategischer wie emergenter „Output" organisationeller Symbolisierungen und Inszenierungen.

Man denke etwa an die in Deutschland (anders als anderswo) klassenübergreifende Niedrigpreisorientierung und Schnäppchenjagd, über die sich Einzelhändler und ihre Verbände schon seit Jahren wortreich beklagen: Es ist schwerlich im langfristigen Interesse eines Unternehmens, eine aggressive Niedrigpreisstrategie zu fahren. Wenn aber um Marktanteile konkurriert wird, macht es Sinn, dies zu tun, ehe es der Wettbewerber tut. Sodann scheitert dieses Einzelhandels-Geschäftsmodell quasi am Erfolg und schließt die Einzelhändler in die gewählte Strategie ein - nicht zuletzt, weil sich entsprechende Kundenerwartungen institutionalisieren. Das Aldi-Shopping der Mittelschichtangehörigen hat bekanntlich in Gestalt von Aldi-Kochbüchern alltagskulturelle Aufwertung erfahren. Standardisierte Dienstleistungen beziehen hier offensichtlich zusätzlich zur reinen Ersparnis noch einen Legitimationsgewinn aus einer Norm sozialer Gleichheit (vgl. Voswinkel 2000).

Im gesellschaftlichen Kontext betrachtet kann man überlegen, ob hier nicht nur bestimmte Marktorientierungen institutionalisiert werden, sondern nebenher auch die normativen Standards an die Akteure auf den Märkten durchgereicht werden, die sich eine Gesellschaft für Gleichheit und Schutz gegen sozialen 
Abstieg setzt. Nachdem die soziale Absicherung auch der Mittelschichten auf der Einkommensseite prekär erscheint, verlagern sich Erwartungen bzw. Bedürfnisse nach Sicherheit und Gleichheit womöglich auf die Konsumseite. Dann ergäbe das Aldishopping in der - vielleicht nicht einmal bewussten Sicht der Kunden ein quasi institutionalisiertes und vielleicht ritualisiertes Auffangnetz gegen Ängste vor dem sozialen Abstieg. Wieweit solche in diesem Fall nicht allein organisationell, sondern sozialstrukturell erzeugten - oder durch unterschiedliche soziale Erfahrungen und Erwartungen überdeterminierten Kundendispositionen aber zur Ressource für den Markterfolg der entsprechenden Einzelhändler werden oder zur Innovationsblockade, das ist fraglich.

\section{Und die Kundin?}

Wer oder was ist nun die Kundin? Sie ist per Definition das Gegenüber einer wirtschaftlichen Transaktion, wobei wir institutionenökonomisch noch nichts über deren Beschaffenheit - einmalig, dauerhaft, relational - gesagt haben. Die Kundin ist jedoch, wenn sie als solche beobachtet und angesprochen wird, individualisierter als der anonyme Markt, und die Beziehung zu ihrem Geschäftspartner dauerhafter. Aber: Sie ist nicht in der Organisation, nicht qua Mitgliedsrolle deren Weisungen unterworfen. Sie ist also ein für die meisten Organisationen und wirtschaftlichen Akteure hoch relevanter Teil ihrer Umwelt, den natürlich diese selbst als solchen konstruieren. Und dieses Konstruieren muss man sich dann einerseits kognitiv, andererseits real vorstellen: Die Kundin als unabhängiger, anderer Marktakteur, den eine Organisation nach Maßgabe ihrer Zwecke beobachtet und auf dessen Anforderungen sie re-agiert zum einen, zum anderen die Kundin, die die Organisation ganz strategisch umwirbt, zu binden und zu verzaubern sucht, die sie also daran zu hindern sucht, sich wie ein Marktakteur zu verhalten - wenn es sich denn machen lässt. Organisationstheoretisch wird also deutlich, dass die Kundin sowohl der handlungsfähige andere Akteur, der „Alter“ der Wirtschaftsorganisation ist und damit der Bezugspunkt von Anpassung der Organisation, als auch das strategisch adressierte Konstrukt der Organisation - und beide theoretische Sichtweisen ergänzen und korrigieren einander, weil sie die beiden Seiten der dialectic of control markieren. In der Tat wird die Gestaltung der Kundenbeziehung Bestandteil und Medium organisationeller Flexibilisierung. 
Wenn Managerinnen also erklären was 'der Kunde' verlangt, kann das beides sein: Beobachtend-kognitive und strategisch-proaktive Konstruktion, enactment oder action - oder gar ein flexibles, im Brunssonschen Sinne heuchlerisches Oszillieren zwischen beidem - der strategischen Suggestion von Beobachtung, wo längst beeinflusst wird oder von Strategie, wo man nur reagieren kann. Ersteres ist in der Darstellung von Organisationen gegenüber der Kundschaft und Öffentlichkeit wahrscheinlicher, zweiteres gegenüber Kapitalgebern. Organisationen sind jedoch diejenigen Akteure, die zentral Kundenbeziehungen nicht nur vollziehen, sondern auch gestalten - und die damit auch erst die Kundin zu einer solchen machen. Vielleicht ist sie doch das Geschöpf der Organisation - wofür sich die Sicht der postmodernen Diskurstheoretiker zumindest als ergänzende Perspektive unter anderen anbietet.

\section{Fazit}

Es sollte deutlich werden, dass die Offenheit und Spezifizierung von Diensten und Leistungen in Wechselwirkungen zwischen Interaktionen, Organisationen und Institutionen erfolgt. Kundenerwartungen sind kulturell und institutionell geprägt, aber sie werden auch in Interaktionen gemäß den Vorgaben der Organisation strategisch beeinflusst. Für das Arbeitshandeln der DienstleisterInnen gilt dasselbe. Damit spielt sich schon die Interaktion in einem Dreieck $\mathrm{ab}$, in dem unterschiedliche Allianzen möglich sind. Jedoch haben Macht, Herrschaft und Kontrolle zwischen Organisationen, AgentInnen und Kunden mehr Ebenen, als die Vorstellung des Kontrolldreiecks zulässt. Vor und nach der Interaktion werden Erwartungen, Präferenzen und normative Orientierungen generiert und institutionalisiert - die mitunter auch in Widersprüche oder Teufelskreise geraten können. Dreiecksbeziehungen finden sich auch zwischen Kunden, Organisationen und der Ebene der Institutionen und der Gesellschaft: Institutionelle Regulierung, mimetische Angleichung oder auch strategische Ausbrüche und Übergriffe von Organisationen bilden hier rekursive Schleifen unter mehr oder weniger öffentlicher Beobachtung; institutionelle Ensembles und kulturelle (Leit-)Bilder werden aktualisiert oder/und transformiert. Wechselseitig, interaktiv, auf mehreren Ebenen zwischen Alltag und Sozialstruktur also werden Dienste und Leistungen, Produkte und Tarife, Pauschalierungen und Differenzierungen ausgehandelt und spezifiziert. In diesem Netz aus Handlungen, Strategien und Wechselwirkungen 
sind jedoch Organisationen - bestimmte fokale Organisationen oder wiederum Netzwerke etc. - besonders mächtige Akteure.

Der strukturationstheoretisch inspirierte Vorschlag, Dienstleistungen in einem Modell mehrerer Ebenen von Interaktionen, Organisationen, Institutionen und Gesellschaften zu erforschen, die durch das Handeln individueller und kollektiver Akteure wechselseitig aktualisiert werden, ist freilich $\mathrm{zu}$ maximalistisch, um in jeder Untersuchung berücksichtigt zu werden. Von DienstleistungsforscherInnen sollte man jedoch erwarten können, dass sie die Aufmerksamkeit auf die Vermittlung zwischen Ebenen richten, sich der Schnittstellen bewusst sind - denn an eben diesen wird in ihrem Untersuchungsfeld gearbeitet. Dann aber ist von der Dienstleistung einiges für das Verständnis gesellschaftlicher Arbeit und Produktion insgesamt zu lernen. Sie zwingt uns ganz unvermeidlich, den Blick auf Prozesse statt Produkte zu richten: auf die soziale Konstruiertheit und die fortlaufende soziale Konstruktion von Erwartungen und Ressourcen, auf die institutionelle Einbettung wirtschaftlichen und organisierten Handelns und deren strategische Aktualisierung, und auf die Wechselwirkungen zwischen sozialem, institutionellem und organisationellem Wandel.

\section{Literatur}

Baethge, Martin/Wilkens, Ingrid (Hrsg.) (2001): Die große Hoffnung für das 21. Jahrhundert? Perspektiven und Strategien für die Entwicklung der Dienstleistungsbeschäftigung. Opladen: Leske

Batt, Rosemary (2000): Strategic segmentation in front-line services: matching customers, employees and human resource systems. International Journal of Human Resource Management 11: $540-561$

Berger, Ulrike/Offe, Claus (1981): Das Rationalisierungsdilemma der Angestelltenarbeit. Arbeitssoziologische Überlegungen zur Erklärung des Status von kaufmännischen Angestellten aus der Eigenschaft ihrer Arbeit als 'Dienstleistungsarbeit'. In: Kocka, Jürgen (Hrsg.) (1981): 39 $-58$

Blutner, Doris/Brose, Hanns-Georg/Holtgrewe, Ursula (2000): Vertriebshandeln und Organisationsstruktur. In: Minssen, Heiner (Hrsg.) (2000): 141 - 162

Boyle, James (2000): Cruel, Mean, or Lavish? Economic Analysis, Price Discrimination and Digital Intellectual Property. In: Vanderbilt Law Review 536: 2007-2039

Brose, Hanns-Georg (1982): Die Vermittlung von sozialen und biographischen Zeitstrukturen. In: Kölner Zeitschrift für Soziologie und Sozialpsychologie Sonderheft 24. 385-407

Bruhn, Manfred/Meffert, Heribert (Hrsg.) (1998): Handbuch Dienstleistungsmanagement. Von der strategischen Konzeption bis zur praktischen Umsetzung. Wiesbaden: Gabler

Brunsson, Nils (1989): The organization of hypocrisy. Talk, decisions and actions in organizations. Chichester u. a.: Wiley

Coleman, James S. (1986): Die asymmetrische Gesellschaft. Vom Aufwachsen mit unpersönlichen Strukturen. Weinheim: Beltz

Coleman, James S. (1991): Grundlagen der Sozialtheorie. München: Oldenbourg 
D'Alessio, Nestor/Oberbeck, Herbert (1998): Vor dem Aufbruch in eine neue Dienstleistungswelt? In: AB/ISF/Inifes/IfS/SOFI (Hrsg.) (1998): 13-61

DiMaggio, Paul J./Powell, Walter W. (1991): The iron cage revisited: Institutional isomorphism and collective rationality. In: Powell/DiMaggio (Hrsg.) (1991): 63 - 88

Fleming, Peter/Spicer, André (2003): Working at a cynical distance: Implications for power, subjectivity and resistance. In: Organization 10. 1. 157-179.

Frenkel, Stephen, et al. (1999): On the front line. Organization of work in the information economy. Ithaca, London: ILR Press

Friedberg, Erhard (1995): Ordnung und Macht. Dynamiken organisierten Handelns. Frankfurt/Main, New York: Campus

Fuller, Linda/Smith, Vicki (1996): Consumers' reports: Management by customers in a changing economy. In: Macdonald, Lynne/Sirianni, Carmen (Hrsg.) (1996): 74-90

Gather, Claudia/Geissler, Birgit/Rerrich, Maria (Hrsg.) (2002): Weltmarkt Privathaushalt. Münster: Westfälisches Dampfboot

Geissler, Birgit (2002): Die Dienstleistungslücke im Haushalt. Der neue Bedarf nach Dienstleistungen und die Handlungslogik der privaten Arbeit. In: Gather, Claudia/Geissler, Birgit/Rerrich, Maria (Hrsg.) (2002): 30-49

Giddens, Anthony (1988): Die Konstitution der Gesellschaft. Grundzüge einer Theorie der Strukturierung. Frankfurt/Main, New York: Campus

Gottschall, Karin (2001): Zwischen tertiärer Krise und tertiärer Zivilisation: Zur sozialwissenschaftlichen Analyse von Dienstleistungsgesellschaften. In: Berliner Journal für Soziologie 11.2. 217-235

Gottschall, Karin/Pfau-Effinger, Birgit (Hrsg.) (2002): Zukunft der Arbeit und Geschlecht. Diskurse, Entwicklungspfade und Reformoptionen im internationalen Vergleich. Opladen: Leske

Hardy, Cynthia/Leiba-O'Sullivan, Sharon (1998): The power behind empowerment: Implications for research and practice. In: human relations 51. 451 - 483

Hassard, John/Parker, Martin (Hrsg.) (1994): Towards a new theory of organizations. London, New York: Routledge

Hasse, Raimund/Krücken, Georg (1999): Neo-Institutionalismus. Bielefeld: transcript

Häußermann, Hartmut/Siebel, Walter (1995): Dienstleistungsgesellschaften. Frankfurt/Main: suhrkamp

Holtgrewe, Ursula/Kerst, Christian (2002a): Call Center: Die Institutionalisierung von Flexibilität. In: Industrielle Beziehungen 9. 2. 186 - 208

Holtgrewe, Ursula/Kerst, Christian (2002b): Zwischen Kundenorientierung und organisatorischer Effizienz - Callcenter als Grenzstellen. In: Soziale Welt 53. 2. 141 - 160.

Holtgrewe, Ursula/Kerst, Christian/Shire, Karen A. (Hrsg.) (2002): Re-Organizing Service Work. Call Centres in Germany and Britain. Aldershot u. a: Ashgate

Holtgrewe, Ursula/Voswinkel, Stephan (2002): Kundenorientierung zwischen Organisationsrationalität und professionellem Eigensinn. In: Sauer, Dieter (Hrsg.) (2002): 99 118

IAB/ISF/Inifes/IfS/SOFI (Hrsg.) (1998): Jahrbuch Sozialwissenschaftliche Technikforschung 1997. Berlin: sigma

Jepperson, R. L. (1991): Institutions, institutional effects and institutionalism. In: Powell/DiMaggio (Hrsg.) (1991): 204 - 231

Knights, David/Morgan, Glenn (1994): Organization theory, consumption and the service sector. In: Hassard, John/Parker, Martin (Hrsg.) (1994): 131 - 152

Kocka, Jürgen (Hrsg.) (1981): Angestellte im europäischen Vergleich. Göttingen: V \& R 
Korczynski, Marek (2002): Call Centre Consumption and the Enchanting Myth of Customer Sovereignty. In: Holtgrewe, Ursula/Kerst, Christian/Shire, Karen A. (Hrsg.) (2002): 163-182

Kühl, Stefan (1995): Wenn die Affen den Zoo regieren. Die Tücken der flachen Hierarchien. Frankfurt/Main, New York: Campus

Leidner, Robin (1996): Rethinking questions of control: Lessons from McDonald's. In: Macdonald, Lynne/Sirianni, Carmen (Hrsg.) (1996): 29-49

Macdonald, C. Lynne/Sirianni, Carmen (Hrsg.) (1996): Working in the service society. Philadelphia: Temple UP

Maleri, Rudolf (1998): Grundlagen der Dienstleistungsproduktion. In: Bruhn, Manfred/Meffert, Heribert (Hrsg.) (1998): 117-139

March, James G./Olsen, Johan P. (1976): Ambiguity and choice in organizations. Bergen: Universitetsforlaget

March, James G./Olsen, Johan P. (1989): Rediscovering Institutions. The Organizational Basis of Politics. New York u.a.

Mayntz, Renate (Hrsg.) (1988): Differenzierung und Verselbständigung: zur Entwicklung gesellschaftlicher Teilsysteme. Frankfurt/Main, New York: Campus

Meyer, John W./Rowan, Brian (1977): Institutionalized Organization: Formal Structure as Myth and Ceremony. In: American Journal of Sociology 83. 2. 340-363

Meyer, Traute (2002): Mehr einfache Dienstleistungen durch Senkung der Arbeitskosten? Warum der Zusammenhang im Wohlfahrtssektor nicht gilt. In: Gottschall, Karin/Pfau-Effinger, Birgit (Hrsg.) (2002): 231-247

Minssen, Heiner (Hrsg.) (2000): Begrenzte Entgrenzungen. Wandlungen von Organisation und Arbeit. Berlin: sigma

Möll, Gerd (2003): Sich die Probleme des Kunden zu Eigen machen - Kundenorientierung, Wissen und Subjektivität in den neuen Beziehungen zwischen Industrie und Handel. In: Schönberger, Klaus/Springer, Stefanie (Hrsg.) (2003): 44-62

Offe, Claus (1984): "Arbeitsgesellschaft". Strukturprobleme und Zukunftsperspektiven. Frankfurt/Main, New York: Campus

Ortmann, Günther (2003): Organisation und Welterschließung. Wiesbaden: Westdeutscher Verlag

Ortmann, Günther/Sydow, Jörg (Hrsg.) (2001): Strategie und Strukturation. Strategisches Management von Unternehmen, Netzwerken und Konzernen. Wiesbaden: Gabler

Ortmann, Günther/Sydow, Jörg/Türk, Klaus (Hrsg.) (1997): Theorien der Organisation. Opladen: Westdeutscher Verlag

Ortmann, Günther/Sydow, Jörg/Windeler, Arnold (1997): Organisation als reflexive Strukturation. In: Ortmann, Günther/Sydow, Jörg/Türk, Klaus (Hrsg.) (1997): 315 - 354

Ortmann, Günther/Zimmer, Marco (2001): Strategisches Management, Recht und Politik. In: Ortmann, Günther/Sydow, Jörg (Hrsg.) (2001): 301-349

Powell, Walter W./DiMaggio, Paul J. (Hrsg.) (1991): The New Institutionalism in Organizational Analysis. Chicago, London: Chicago UP

Quack, Sigrid/Morgan, Glenn/Whitley, Richard (Hrsg.) (2000): National Capitalisms, Global Competition and Economic Performance. Amsterdam, Philadelphia: John Benjamins

Regini, Mario/Kitay, Jim/Baethge, Martin (Hrsg.) (1999): From Tellers to Sellers. Changing Employment Relations in Banks. Cambridge, Mass., London: MIT Press

Rieder, Kerstin (2001): Empowerment and utilisation of the customer in service work. Beitrag zum Workshop "Personnel, Organization, Poststructuralism" am 1./2. Juni 2001 in Innsbruck. http://iol.uibk.ac.at/conference/abstracts_innsbruck.pdf, Download 2.1.2002).

Ritzer, George (1996): The McDonaldization of Society. An Investigation into the Changing Character of Contemporary Social Life. Thousand Oaks, Calif.: Pine Forge Press 
Rudolph, Hedwig (Hrsg.) (2001): Aldi oder Arkaden? Unternehmen und Arbeit im europäischen Einzelhandel. Berlin: sigma

Sauer, Dieter (Hrsg.) (2002): Dienst - Leistung(s) - Arbeit: Kundenorientierung und Leistung in tertiären Organisationen. München: ISF

Schönberger, Klaus/Springer, Stefanie (Hrsg.) (2003): Technisierung und Subjektivierung - Zur Veränderung des Arbeitshandelns in der Wissensgesellschaft. Frankfurt/Main, New York: Campus

Schreyögg, Georg/Sydow, Jörg (Hrsg.) (1997): Managementforschung 7: Gestaltung von Organisationsgrenzen. Berlin, New York: de Gruyter

Scott, W. Richard/Meyer, John W. (Hrsg.) (1994): Institutional environments and organizations. Structural complexity and individualism. Thousand Oaks u. a.: Sage

Simon, Herbert I. (1957): Models of man. Social and rational. New York

Stichweh, Rudolf (1988): Inklusion in Funktionssysteme der modernen Gesellschaft. In: Mayntz, Renate (Hrsg.) (1988): 261-293

Tacke, Veronika (1997): Systemrationalisierung an ihren Grenzen. Organisationsgrenzen und Funktionen von Grenzstellen in Wirtschaftsorganisationen. In: Schreyögg, Georg/Sydow, Jörg (Hrsg.) (1997): 1 - 44

Thompson, J. D. (1967): Organizations in Action. New York et al.: McGraw-Hil

Türk, Klaus (1997): Organisation als Institution der kapitalistischen Gesellschaftsformation. In: Ortmann, Günther/Sydow, Jörg/Türk, Klaus (Hrsg.) (1997): 124 - 176

Veblen, Thorstein (1986): Theorie der feinen Leute. Frankfurt/Main: Fischer

Voswinkel, Stephan (2000): Das mcdonaldistische Produktionsmodell - Schnittstellenmanagement interaktiver Dienstleistungsarbeit. In: Minssen, Heiner (Hrsg.) (2000): 177 - 201

Weihrich, Margit/Dunkel, Wolfgang (2003): Abstimmungsprobleme in Dienstleistungsbeziehungen. Ein handlungstheoretischer Zugang. In: Kölner Zeitschrift für Soziologie und Sozialpsychologie 55. 4. $758-781$ 
1 Zentrale Arbeiten finden sich in den Sammelbänden von Powell/DiMaggio 1991; Scott/Meyer 1994. Überblicksdarstellungen sind Türk 1997; Hasse/Krücken 1999.

2 Informativ zur Preisgestaltung auf bestimmten Dienstleistungsmärkten ist Boyle 2000.

3 Die Wechselwirkungen von Dienstleistungsgestaltung und Arbeitsmärkten ist immer wieder Gegenstand der Debatten um high-road- oder lowroad-Strategien (d'Alessio/Oberbeck 1998) im Dienstleistungssektor. Die Annahme einer quasi „natürlichen“ Zusammenhangs von hoher Dienstleistungsqualität, Kundenzufriedenheit und Kundenbindung mit ökonomischem Erfolg und mit anspruchsvoller, qualifizierter und abgesicherter Arbeit wird, kann mitunter empirisch belegt werden (Batt 2000). Sie fungiert aber, ob zutreffend oder nicht, auch als Legitimationsressource für Gewerkschaften, Verbraucherlobbies und betriebliche Interessenvertretungen. 